\title{
Synergistic suppression effect on tumor growth of ovarian cancer by combining cisplatin with a manganese superoxide dismutase-armed oncolytic adenovirus
}

\author{
This article was published in the following Dove Press journal: \\ OncoTargets and Therapy \\ 17 October 2016 \\ Number of times this article has been viewed
}

\author{
Shibing Wang ${ }^{1,2, *}$ \\ Jing Shu ${ }^{3, *}$ \\ Li Chen ${ }^{4}$ \\ Xiaopan Chen ${ }^{3}$ \\ Jianhong Zhao ${ }^{4}$ \\ Shuangshuang $\mathrm{Li}^{1,2}$ \\ Xiaozhou Mou ${ }^{1,2}$ \\ Xiangmin Tong ${ }^{1,2}$ \\ 'Clinical Research Institute, Zhejiang \\ Provincial People's Hospital, ${ }^{2}$ Key \\ Laboratory of Cancer Molecular \\ Diagnosis and Individualized Therapy \\ of Zhejiang Province, ${ }^{3}$ Department \\ of Reproductive Endocrinology, \\ Zhejiang Provincial People's Hospital, \\ ${ }^{4}$ Department of Obstetrics and \\ Gynecology, Hangzhou Red Cross \\ Hospital, Hangzhou, People's Republic \\ of China \\ *These authors contributed \\ equally to this work
}

\footnotetext{
Correspondence: Xiangmin Tong Clinical Research Institute, Zhejiang Provincial People's Hospital, 158 Shangtang Road, Hangzhou 3100I4, People's Republic of China $\mathrm{Tel} / \mathrm{fax}+86$ 57/ 858930I2 Email tongxiangmin@163.com
}

\begin{abstract}
Gene therapy on the basis of oncolytic adenovirus is a novel approach for human cancer therapeutics. We aim to investigate whether it will synergistically reinforce their antiovarian cancer activities when the combined use of ZD55-manganese superoxide dismutase (MnSOD) and cisplatin was performed. The experiments in vitro showed that ZD55-MnSOD enhances cisplatin-induced apoptosis and causes remarkable ovarian cancer cell death. Apoptosis induction by treatment with ZD55-MnSOD and/or cisplatin was detected in SKOV-3 by apoptotic cell staining, flow cytometry, and western blot analysis. In addition, the cytotoxicity caused by ZD55-MnSOD to normal cells was examined by the 3-(4,5-dimethyl-2-thiazolyl)-2,5diphenyl-2-H-tetrazolium bromide assay and western blot analysis. Animal experiment further confirmed that combination of ZD55-MnSOD and cisplatin achieved significant inhibition of SKOV-3 ovarian tumor xenografted growth. In summary, we have demonstrated that ZD55MnSOD can sensitize human ovarian cancer cells to cisplatin-induced cell death and apoptosis in vitro and in vivo. These findings indicate that the combined treatment with ZD55-MnSOD and cisplatin could represent a rational approach for antiovarian cancer therapy.
\end{abstract}

Keywords: oncolytic adenovirus, MnSOD, cisplatin, ovarian cancer

\section{Introduction}

Ovarian cancer is the most lethal form of gynecological cancer worldwide and the 5 -year survival rate has remained relatively consistent over the past few decades. ${ }^{1}$ Advanced ovarian cancers are difficult to treat with the currently available chemotherapeutic regimen. Although therapeutic regimens that combine platinum-based therapy with other cytotoxic agents are the current standard of care for advanced ovarian cancer patients, the cumulative toxicities of cisplatin and carboplatin can present barriers for the long-term use of these agents. Therefore, novel methods of therapy devoid of side effects are urgently needed.

Cancer virotherapy is a tumor-specific therapeutic strategy, in which viruses are engineered to preferentially replicate in tumor cells and destroy it by lysis, either through targeted alterations in the cancer cells, such as p53 mutation, viral deletion, tissue-specific transcriptional control, or tumor-specific receptors. ${ }^{2}$ Among available virotherapies, conditionally replicating adenovirus is attractive because of its several attributes, including lytic replication, efficient genome transfer, and excellent patient safety in clinical trials. ${ }^{3,4}$ In our previous study, we have developed a cancer-targeting gene virotherapy strategy ${ }^{5}$ and generated a novel E1B55K gene deleted oncolytic 
adenovirus ZD55-MnSOD, harboring manganese superoxide dismutase (MnSOD) gene. ${ }^{6}$ Our data have showed that the overexpression of MnSOD in colorectal cancer causes remarkable cancer cell death both in vitro and in vivo, and the production of $\mathrm{H}_{2} \mathrm{O}_{2}$ derived from MnSOD dismutationactivated caspase- 8 , which might downregulate Bcl-2 expression and cause Bax translocation to mitochondria.

MnSOD is located in the mitochondrial matrix, which converts $\mathrm{O}_{2}-$ to $\mathrm{H}_{2} \mathrm{O}_{2}$ and $\mathrm{O}_{2}$. MnSOD is an antioxidant enzyme with tumor suppressor activity, whereas the molecular mechanisms of its antitumor effects remain unclear. Some studies have shown that overexpression of MnSOD inhibits the growth of numerous tumor cell types. ${ }^{7}$ Growing evidence supports the antitumor effects of MnSOD gene therapy in the models of colorectal, pancreatic, breast, and prostate cancers. $^{8-10}$ To our knowledge, there is no report available of studies exploring the replication-competent recombinant adenovirus-mediated MnSOD gene therapy in ovarian cancer.

In consideration of the severe damage and high lethality of ovarian cancer, the strategy of chemo-geneviro-therapy of combining cisplatin with ZD55-MnSOD was used. Our previous studies have demonstrated that the combination of ZD55 armed tumor necrosis factor-related apoptosisinducing ligand (TRAIL), second mitochondria-derived activator of caspases (Smac), XIAP associated factor 1 (XAF1), or interleukin 24 (IL-24) gene, and cisplatin obviously enhanced antitumor effects and reduced side effects. ${ }^{11-14}$ In this article, we report the antitumor effect of ZD55-MnSOD and its combination with cisplatin, and explore the possible mechanisms underlying the action of MnSOD and cisplatin in both ovarian cancer cell line SKOV-3 and an animal model of ovarian tumor xenograft.

\section{Materials and methods}

\section{Cell lines and viruses}

The human ovarian cancer cell lines SKOV-3 were obtained from the Cell Bank of Type Culture Collection of Chinese Academy of Sciences (Shanghai, People's Republic of China) and cultured in Dulbecco's Modified Eagle's Medium (GIBCO, Carlsbad, CA, USA) supplemented with $10 \%$ heat inactivated fetal bovine serum (GIBCO). Cells were incubated in a $5 \% \mathrm{CO}_{2}$ humidified incubator at $37^{\circ} \mathrm{C}$. Construction and production of recombinant oncolytic adenovirus ZD55-MnSOD were described previously. ${ }^{6}$ MnSOD cDNA was transcribed by reverse transcriptionpolymerase chain reaction (PCR) from human fetal liver cells (LO2), and the MnSOD sequence from this cDNA was further amplified by PCR. The amplification was done using forward (5'-CGGAATTCATGAAGCACAGCCTCCCC-3') and reverse $\left(5^{\prime}\right.$-TGCTCTAGAGCATAACGATCGTGGT TTAC-3') primers. The MnSOD gene (excised by EcoRI/ XbaI) from pBlueScript (+)-MnSOD was cloned into pCA13, which was excised by EcoRI/XbaI aforehand to construct pCA13-MnSOD. The pZD55-MnSOD was constructed by inserting the whole foreign gene expression cassette cut from pCA13-MnSOD using BglII into the corresponding site of pZD55. All plasmid constructs were confirmed by restrictive enzyme digestion, PCR, and DNA sequence. The amplification of recombinant adenovirus was amplified by infecting 293A cells.

\section{Cytotoxicity assay and quantitative analysis of synergy in vitro}

SKOV-3 cells were seeded into 96-well plates at a density of $1 \times 10^{4}$ in $100 \mu \mathrm{L}$ culture medium. Cells were treated with different treatments of indicated concentrations. After 48 or 72 hours, 3-(4,5-dimethyl-2-thiazolyl)-2,5diphenyl-2-H-tetrazolium bromide (MTT, Sigma-Aldrich, St Louis, MO, USA) solution ( $10 \mu \mathrm{L}, 5 \mathrm{~g} / \mathrm{L})$ was added to the cells and then cultivated for a further 4 hours. A total of $570 \mathrm{~nm}$ absorbance was measured using a DNA microplate reader (GENios model; Tecan; Maennedorf, Switzerland).

\section{Reactive oxygen species assay}

Intracellular reactive oxygen species generation was assessed by the reactive oxygen species assay kit. Cells were infected either with a single drug or combination therapy. During the last 30 minutes of incubation, $10 \mu \mathrm{M} 2,7$-dichlorodihydrofluorescein diacetate (DCFH-DA) was applied to the cells. After quickly detaching from the plate, the cells were immediately subjected to flow cytometry. DCF-positive cells were counted as an indicator of $\mathrm{H}_{2} \mathrm{O}_{2}$ generation.

\section{Hoechst 33342 staining assay}

Hoechst 33342 staining was used to detect morphological features of cell apoptosis. SKOV-3 cells were treated with cisplatin or ZD55-MnSOD, or the combination of cisplatin and ZD55-MnSOD. After treating for 72 hours, $1 \mathrm{mg} / \mathrm{mL}$ (5 $\mu \mathrm{L}$ ) Hochest 33342 (Sigma-Aldrich) was added to the cells for 30 minutes and the results were observed under inverted fluorescence microscope. Untreated cells served as a control.

\section{Flow cytometry analysis}

SKOV-3 cells were treated with cisplatin $(4 \mu \mathrm{g} / \mathrm{mL})$, ZD55MnSOD (10 multiplicity of infection [MOI]), or cisplatin (4 MOI) plus ZD55-MnSOD (4 $\mu \mathrm{g} / \mathrm{mL})$. After 48 hours, apoptosis cells were detected by using Annexin V-fluorescein 
isothiocyanate (FITC) and propidium iodide (PI) double staining or PI staining alone following the manufacturer's introduction. Cell apoptosis and cell cycle were examined using FACS (FACStar cytofluorometer; BD Biosciences, San Jose, CA, USA).

\section{Western blot analysis}

SKOV-3 cells were collected and washed twice with phosphate-buffered saline (PBS), then lysed in radioimmunoprecipitation assay buffer. Proteins were separated by $12 \%$ sodium dodecyl sulfate polyacrylamide gel and transferred to polyvinylidene fluoride membrane. Then the membrane was incubated with primary antibodies and dilutions used were adenovirus-5 E1A $(1: 1,000)$, MnSOD $(1: 1,000)$, caspase- 8 $(1: 1,000)$, caspase-3 $(1: 1,000)$, poly (ADP-ribose) polymerase (PARP; $1: 1,000)$, and $\beta$-actin $(1: 1,000)$. The secondary antibodies used were anti-rabbit $(1: 5,000)$ and anti-mouse (1:5,000). Antibodies against adenovirus-5 E1A, MnSOD, caspase-8, caspase-3, PARP, and $\beta$-actin were purchased from Santa Cruz Biotechnology (Santa Cruz, CA, USA).

\section{Animal experiments}

All animal experiments were approved by the Institutional Animal Care and Use Committee, performed according to the Guide for the Care and Use of Laboratory Animals of the National Institutes of Health, and followed the guidelines of the Animal Welfare Act. Female BALB/c nude mice (4-5 weeks old) were purchased from Shanghai Experimental Animal Center (Shanghai, People's Republic of China). The SKOV-3 cells were injected subcutaneously into the lower right flank of female nude mice and tumor xenografts model was established. Each group was composed of at least eight animals and tumor growth was monitored and measured for every 5 days with a Vernier caliper. Tumor volume (V) was calculated according to the formula: $\mathrm{V}\left(\mathrm{mm}^{3}\right)=1 / 2 \times$ length $(\mathrm{mm}) \times$ width $(\mathrm{mm})^{2}$. Once the subcutaneous tumors reached $\sim 100-150 \mathrm{~mm}^{3}$, the nude mice were divided into four groups ( 8 mice in each group) randomly. Subsequently, mice were injected with cisplatin, ZD55-MnSOD, combination of the virus and the drug, or PBS. ZD55-MnSOD $\left(1 \times 10^{9}\right.$ plaque forming unit/mouse) was injected once every other day for a total of three times through intratumoral injection; cisplatin was injected intraperitoneally at a total dose of $5 \mathrm{mg} / \mathrm{kg}$ body weight; and PBS $100 \mu \mathrm{L}$ as control for a total of three times once every other day.

\section{Statistical analysis}

The experimental statistical significance was expressed as mean \pm standard error (SE) and analyzed by Graph Pad 6.0 software and Student's or paired $t$-test. When $P<0.05$, it is considered statistically significant.

\section{Results \\ Potent antitumor activity of ZD55- MnSOD in ovarian cancer cell}

The structures of the recombinant adenoviruses constructed for this study are presented in Figure 1A. In ovarian cancer cell line (SKOV-3), infection with ZD55-MnSOD and ZD55 at the indicated MOIs resulted in significant production of adenovirus protein E1A. To assess the transgenic delivery ability mediated by ZD55, SKOV-3 cells were treated with ZD55-MnSOD or ZD55 for 48 hours, and western blot assay was performed to detect the expression of MnSOD gene. As shown in Figure 1B, MnSOD was expressed efficiently in SKOV-3 cells. In human normal liver cell line (QSG-7701), no obvious expression of E1A was detected after treatment with ZD55-MnSOD or ZD55 (Figure 1D). These results indicated that ZD55-MnSOD had cancer cell-selective replication properties, and MnSOD transgene was effectively expressed.

To investigate the effect of ZD55-MnSOD on cell viability, and also to confirm the toxicity results, the human ovarian cancer cell SKOV-3 and human normal cell QSG-7701 were infected with ZD55-MnSOD and ZD55 at a MOI of 10, respectively. Cell proliferation was measured by MTT assay and the results showed that ZD55-MnSOD induced cell death in $70.2 \%$ of the infected cancer cell lines within 4 days postinfection and ZD55-MnSOD was clearly more effective than ZD55 in killing cancer cells (Figure 1C). In contrast, $92.7 \%$ of normal human cells were still viable at that dose of virus (Figure 1E).

\section{ZD55-MnSOD enhances cisplatin- mediated growth inhibition in ovarian cancer cells}

To assess whether the ZD55-MnSOD enhances cisplatininduced cell death in ovarian cancer cells, MTT assay was performed as mentioned previously. Ovarian cancer cell line SKOV-3 was infected with cisplatin, ZD55-MnSOD, or combination treatment. The results suggest that the combination of cisplatin and ZD55-MnSOD has an enhanced tumor killing effect on ovarian cancer (Figure 2A). Next, synergistic effect of cisplatin combined with ZD55-MnSOD on ovarian cancer cell SKOV-3 was quantified by combination index (CIN) analysis and expressed as CIN versus fraction affected in Figure 2B. These results showed that the combination of cisplatin and ZD55-MnSOD has an enhanced tumor killing effect. 

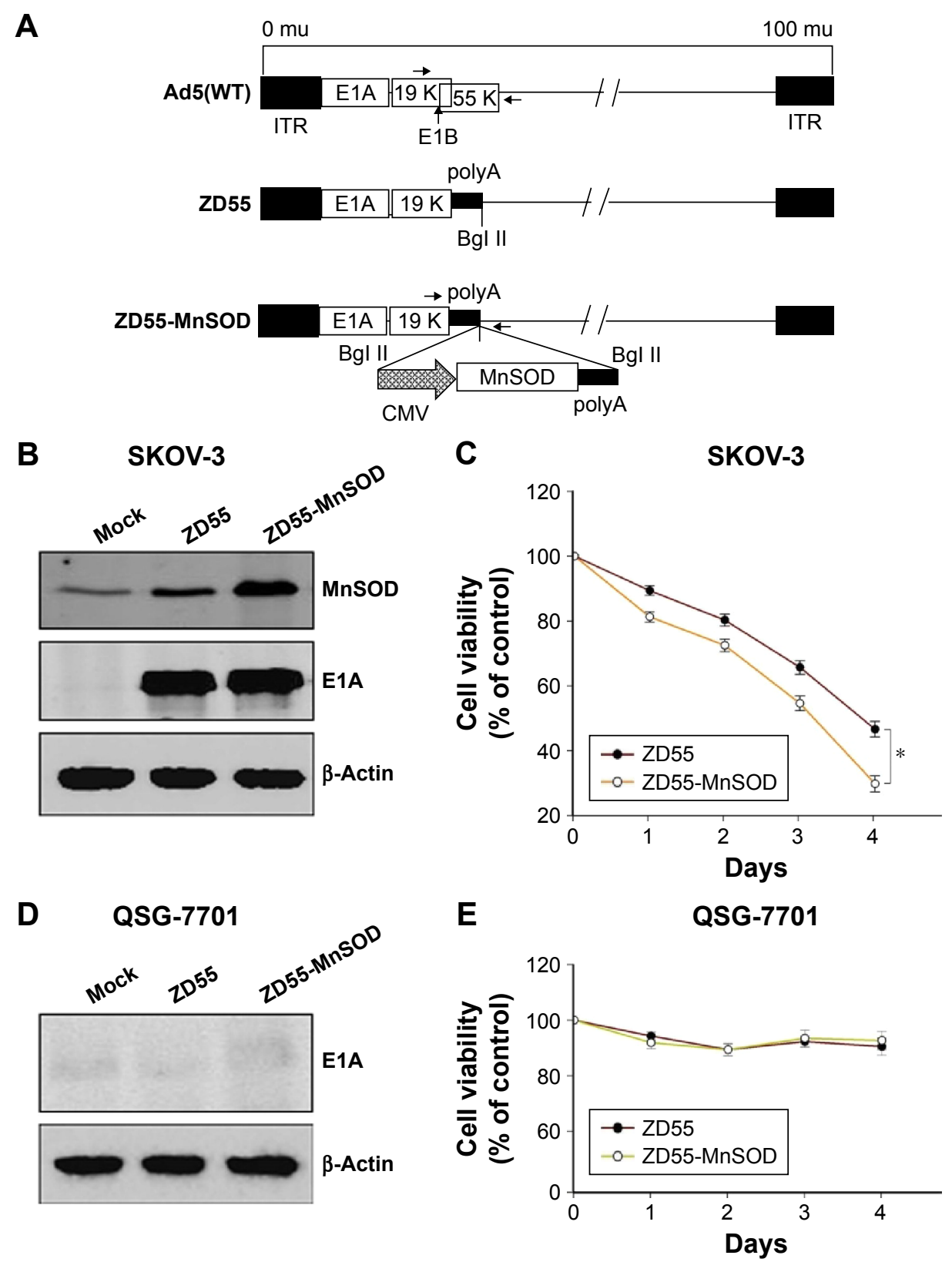

Figure I Characterization of ZD55-MnSOD and its selective replication in ovarian cancer cells.

Notes: (A) Schematic structure of Ad5(WT), ZD55, and ZD55-MnSOD. In ZD55-MnSOD, the EIB 55-kDa gene (bp2269-3327) was replaced by SV40 polyA and the expression box of MnSOD. (B) Detection of expression of EIA and MnSOD by western blot analysis. SKOV-3 cells were treated with ZD55 or ZD55-MnSOD at a MOI of I0. After 48 hours, the cell lysates were prepared for analyzing the expression of MnSOD and EIA proteins. Mock-infected cells were included as a control. $\beta$-Actin was used as a protein loading control. (D) EIA protein expression in human normal liver cells (QSG-770I) was detected by western blotting after treatment with ZD55 or ZD55-MnSOD at a dose described above. Ovarian cancer cell line SKOV-3 (C) and human normal cell lines QSG-770I (E) were infected with ZD55 and ZD55-MnSOD at a MOI of 5, respectively. On days I, 2, 3, and 4 postinfection, cell viability was examined by MTT colorimetric assay. Data shown (mean \pm standard error) are representative of three experiments. $* P<0.05$.

Abbreviations: MnSOD, manganese superoxide dismutase; WT, wild type.

To verify whether the enhanced MnSOD expression can enrich the $\mathrm{H}_{2} \mathrm{O}_{2}$, a $\mathrm{H}_{2} \mathrm{O}_{2}$-sensitive probe DCFH-DA was used to detect $\mathrm{H}_{2} \mathrm{O}_{2}$ generation. The result shows that there was a significant increase in the percentage of DCF-positive tumor cells infected with ZD55-MnSOD compared with the uninfected cell $(P<0.01)$, and this effect appears remarkable when the cells are exposed to ZD55-MnSOD combined with cisplatin. Moreover, the combination treatment is more effective than ZD55-MnSOD $(P<0.05)$ and cisplatin $(P<0.01)$ (Figure 2C). It is well known that $\mathrm{O}_{2}-$ can be dismuted to $\mathrm{H}_{2} \mathrm{O}_{2}$ and $\mathrm{O}_{2}$ by MnSOD expression. Thus, these results indirectly suggest that either the overexpression of MnSOD or cisplatin treatment led to the accumulation of $\mathrm{H}_{2} \mathrm{O}_{2}$, as evidenced by the raised percentage of DCF-positive cells. 

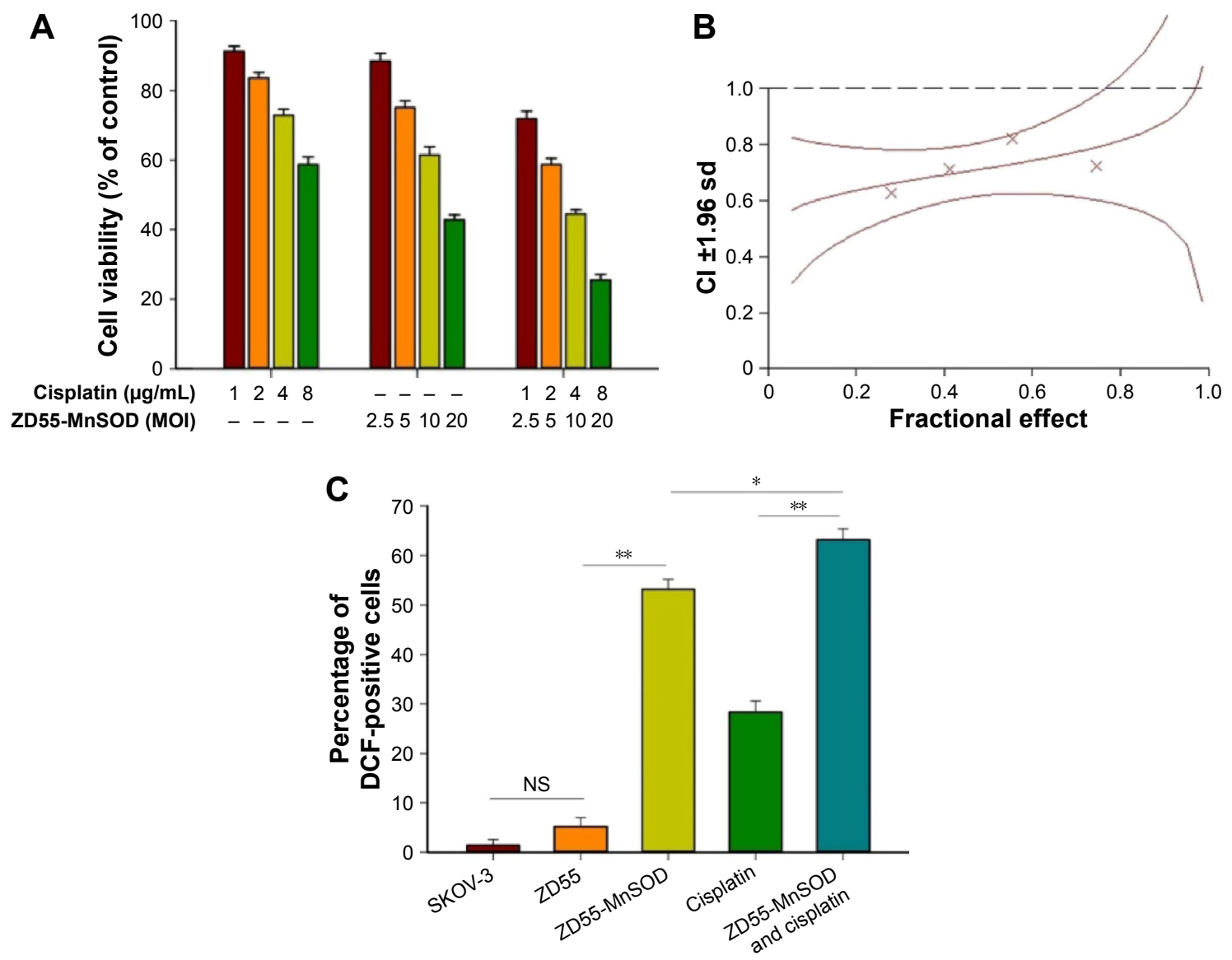

Figure 2 ZD55-MnSOD enhances cisplatin-mediated growth inhibition in ovarian cancer cells.

Notes: (A) Ovarian cancer cells SKOV-3 were treated with cisplatin (I, 2, 4, $8 \mu \mathrm{g} / \mathrm{mL})$, ZD55-MnSOD (2.5, 5, I0, 20 MOI), or combination for 48 hours. The image showed three independent experiments respectively. Cell viability was evaluated by MTT assay. (B) Synergistic effect of cisplatin combined with ZD55-MnSOD on SKOV-3. It was quantified by $\mathrm{CIN}$ analysis and expressed as CIN versus fraction affected. Where calculable, $95 \%$ confidence intervals are shown. (C) ROS generation in SKOV-3 cells. Cells were infected with ZD55 (I0 MOI), ZD55-MnSOD (I0 MOI), cisplatin $(4 \mu \mathrm{g} / \mathrm{mL})$, or cisplatin $(4 \mu \mathrm{g} / \mathrm{mL})$ plus ZD55-MnSOD (I0 MOI). Forty-eight hours later, cells were incubated with DCFH-DA for 30 minutes in the dark before harvest. Cell suspension was subjected to flow cytometric analysis. NS, $P>0.05$; $* P<0.05$; $* * P<0.0$ I.

Abbreviations: MnSOD, manganese superoxide dismutase; MTT, 3-(4,5-dimethyl-2-thiazolyl)-2,5-diphenyl-2-H-tetrazolium bromide; CIN, combination index; ROS, reactive oxygen species; DCFH-DA, 2, 7-dichlorodihydrofluorescein diacetate.

\section{ZD55-MnSOD enhances cisplatin- mediated apoptosis in ovarian cancer cells}

Subsequently, Hoechst 33342 staining was designed to investigate the morphological alterations of SKOV-3 cells treated with cisplatin plus ZD55-MnSOD, cisplatin, or ZD55MnSOD alone. The results demonstrated that compared with treatment of cisplatin alone, co-treatment with ZD55MnSOD led to marked apoptosis, characterized by chromatin condensation, nuclear fragmentation, and apoptotic bodies. The number of apoptotic cells is quantitated in Figure 3A. To quantify the effects of ZD55-MnSOD on cisplatin-induced apoptosis, Annexin-V-FITC/PI double staining was used to analyze cell apoptosis (Figure 3B). The results demonstrated that the apoptotic rate of SKOV-3 cells co-treated with cisplatin and ZD55-MnSOD was $37.9 \%$, which is nearly three times more than that of cisplatin treatment alone (12.8\%).
Western blot analysis demonstrated that cisplatin has the capacity to activate the caspase-dependent pathway, including activation of caspase-8, caspase- 3 , and cleavage of PARP, and this unique effect can be further enhanced by cotreatment with cisplatin and ZD55-MnSOD (Figure 3C).

\section{ZD55-MnSOD enhances cisplatin- mediated ovarian tumor growth suppression in vivo}

To test the therapeutic effects of combination of cisplatin and ZD55-MnSOD in vivo, animal experiments were performed using an ovarian tumor xenograft model established by SKOV-3 cells. Tumor growth curves were plotted to compare the difference of their antitumor efficacy during a 55-day observation. As shown in Figure 4A, there was a significant decrease in mean tumor volume in animals receiving intratumoral injections of cisplatin, ZD55-MnSOD, and 
A

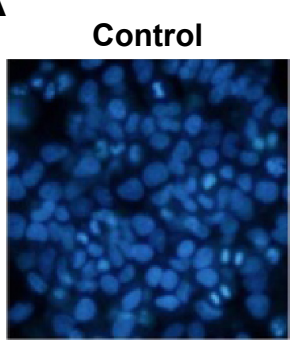

\section{Cisplatin}

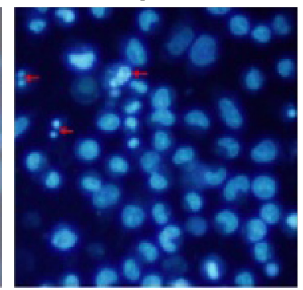

\section{ZD55-MnSOD}

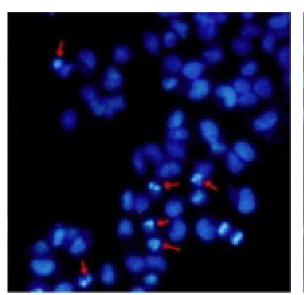

ZD55-MnSOD and cisplatin

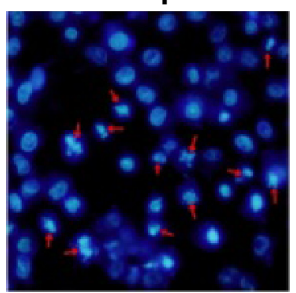

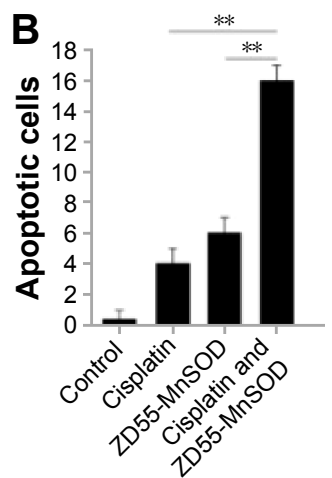

B

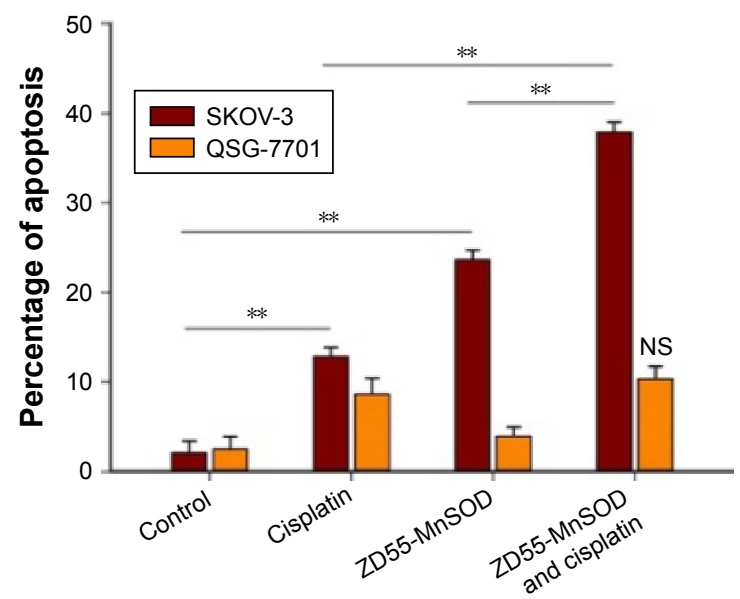

C

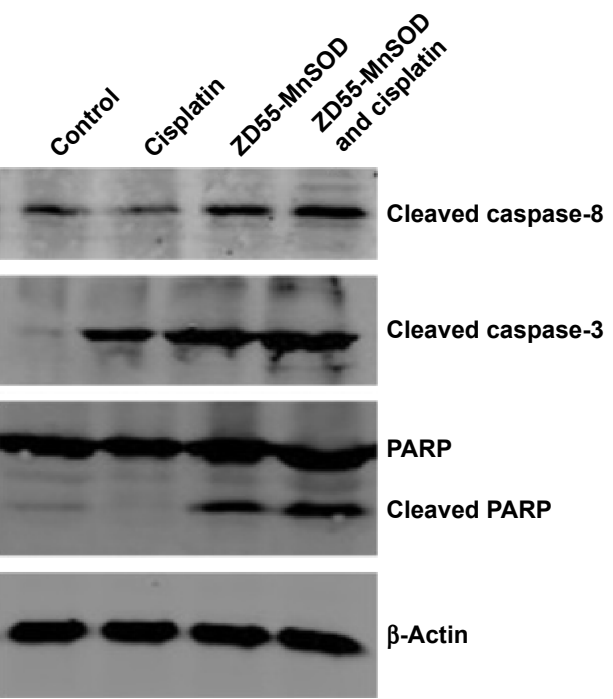

Figure 3 ZD55-MnSOD enhances cisplatin-induced apoptosis in ovarian cancer cells.

Notes: (A) Hoechst 33342 staining was used to detect apoptosis. SKOV-3 cells were seeded in 96-well plates and infected with cisplatin (4 $\mu$ g/mL), ZD55-MnSOD (I0 MOI), or cisplatin $(4 \mu \mathrm{g} / \mathrm{mL}$ ) plus ZD55-MnSOD (I0 MOI). Seventy-two hours later, SKOV-3 cells were treated with Hoechst $333425 \mu \mathrm{L}$ (I mg/mL) for $30 \mathrm{minutes}$ and then observed under the inverted fluorescence microscope. The red arrows indicate positive apoptotic cells. The number of apoptotic cells are quantitated in histogram. Original magnification, $\times 200$. (B) Cisplatin $(4 \mu \mathrm{g} / \mathrm{mL})$, ZD55-MnSOD (I0 MOI), or cisplatin $(4 \mu \mathrm{g} / \mathrm{mL})$ plus ZD55-MnSOD (I0 MOI) was used to treat SKOV-3 and QSG770I. Uninfected cells served as control. Forty-eight hours later, apoptosis was determined by flow cytometry. NS, $P>0.05$; $* * P<0.0$ I. (C) SKOV-3 cells were infected with cisplatin $(4 \mu \mathrm{g} / \mathrm{mL})$, ZD55-MnSOD (IO MOI), or cisplatin $(4 \mu \mathrm{g} / \mathrm{mL})$ plus ZD55-MnSOD (IO MOI) for 48 hours. Whole cell extracts were prepared and immunoblotted for the detection of activation of caspase pathway. $\beta$-Actin was used as a loading control.

Abbreviations: MnSOD, manganese superoxide dismutase; PARP, poly (ADP-ribose) polymerase; NS, not significant.
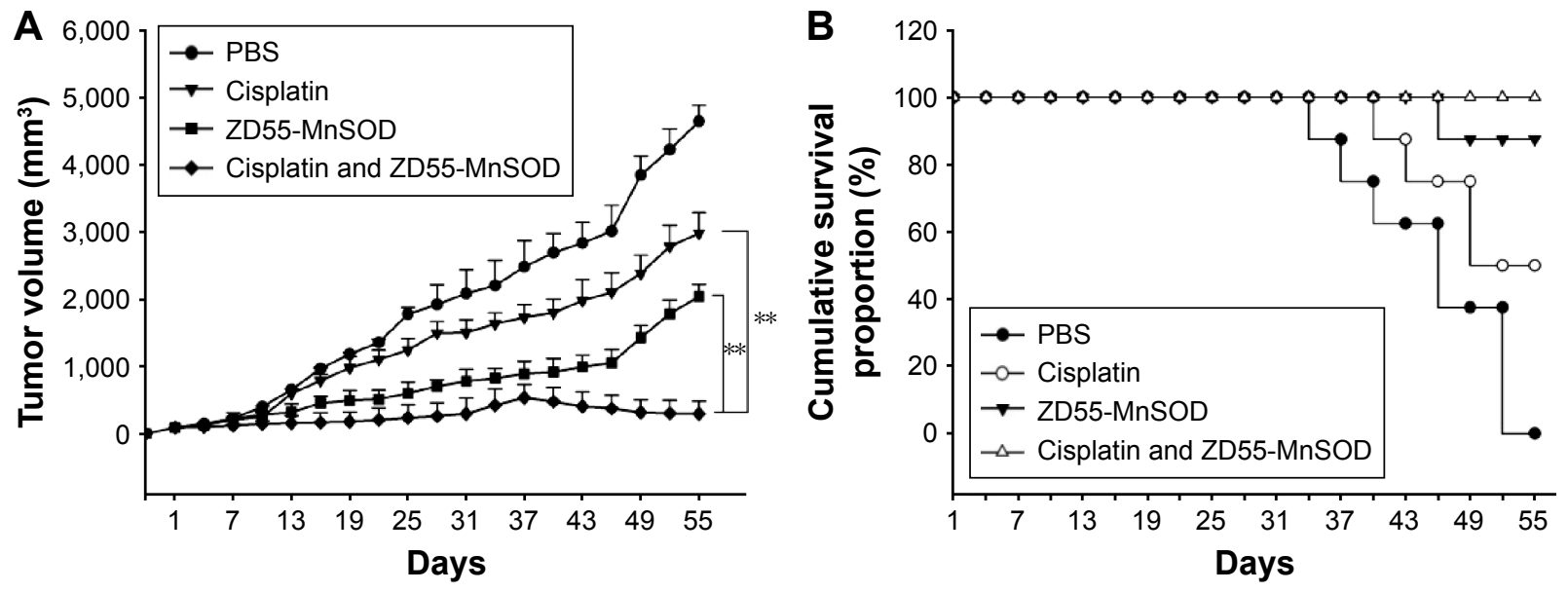

Figure 4 Synergistic effects of cisplatin and ZD55-MnSOD in vivo.

Notes: (A) Tumor volume was measured at different times after treatment. Data are presented as mean \pm standard error $(\mathrm{n}=6)$. $* * P<0.01$. (B) The image shows inhibitory effect of each group on tumor growth at the last time point (day 55) when the mice were sacrificed.

Abbreviations: MnSOD, manganese superoxide dismutase; PBS, phosphate-buffered saline. 
their combination compared with those receiving injections of PBS. As expected, the combination treatment was more effective than cisplatin $(P=0.002)$ and ZD55-MnSOD alone ( $P=0.002)$. Moreover, the combination therapy of cisplatin and ZD55-MnSOD resulted in an improved survival rate compared with PBS, cisplatin or ZD55-MnSOD alone (Figure 4B).

\section{Discussion}

Monotherapy of cancer often has limited success, as the tumor cells usually develop resistance to the agents used and tumors are usually genetically diverse. ${ }^{15,16}$ Therefore, it is important to identify combinations of two or more therapeutic agents, acting through different mechanisms with synergistic effects without increasing adverse effects. Several clinical studies have illustrated that combination therapy of oncolytic adenovirus and chemotherapy or radiation therapy exerts synergistic antitumor activity. ${ }^{17-20}$ It also showed that the way of oncolytic adenoviruses combined with cytotoxic chemotherapies will enhance the potential of oncolytic adenovirus and optimize treatment.

Although chemotherapy drugs, such as cisplatin, are of significant importance in the treatment of various malignant tumors, drug resistance and side effects are the major clinical obstacles. ${ }^{21}$ Several combined drug administration schemes have been successfully applied with potent antitumor efficacy and slight adverse effects. However, the combination of cisplatin and ONYX-015 has been reported to have no synergistic toxicities. ${ }^{22}$ Thus, the combination strategies should be improved to apply in clinical cancer therapy.

In this study, we adopted a new chemo-geneviro-therapy strategy, which combined the advantage of MnSOD-armed oncolytic adenovirus and cisplatin. Our aims were to confirm the enhanced antitumor effect of ZD55-MnSOD with cisplatin in ovarian cancer cells in vitro and in vivo, and to assess the cytotoxicity on normal liver cells. The results suggested that ZD55-MnSOD could not only replicate in ovarian cancer cells, but also express MnSOD gene efficiently in ovarian cancer cells (Figure 1), leading to enhanced inhibition on the proliferation of ovarian cancer cells by combining cisplatin (Figure 2).

Apoptosis is the important antitumor mechanism in various treatment strategies, including cancer targeting genevirotherapy and chemotherapy. ${ }^{6}$ Our data demonstrated that, compared with treatment of cisplatin alone, co-treatment with ZD55-MnSOD led to marked apoptosis characterized by chromatin condensation, nuclear fragmentation, and apoptotic bodies. The ZD55-MnSOD has been reported to trigger cell apoptosis in a caspase-independent manner. However, the present study confirmed that the caspase apoptotic pathway is involved in the antitumor process, and caspase-8, caspase-3, and PARP cleavage were observed in ovarian cancer SKOV-3 cells with ZD55-MnSOD treatment and its combination with cisplatin (Figure 3). The combination showed the outstanding increase in caspase-8, caspase-3, and PARP cleavage compared with other groups.

MnSOD is a kind of antioxidant enzyme in mitochondria, which converts superoxide into hydrogen peroxide, and plays an important protective role against oxidative stress. Overexpression of MnSOD protects cells and transgenic animals from various forms of oxidative stress. In addition, increased levels of MnSOD have been shown to reduce cell growth and induce differentiation. The present study investigated the possibility of combining cisplatin with ZD55-MnSOD, a MnSOD-armed oncolytic adenovirus that may be a novel therapeutic agent for patients with ovarian carcinoma, in order to reinforce their antitumor activities.

In summary, we have demonstrated for the first time, to the best of our knowledge, that ZD55-MnSOD sensitizes human ovarian cancer cells to cisplatin-induced cell death in vitro and in vivo. These findings indicate that combined treatment with cisplatin and ZD55-MnSOD could represent a rational approach for antiovarian cancer therapy.

\section{Acknowledgments}

This article was supported by Science and Technology Research Foundation of Hangzhou (No 20140733Q24) to LC, the Zhejiang Provincial Natural Science Foundation of China (Nos LY13H080005, LY15H160051, and LY14H160041), National Science Foundation of China (No 81570198 to XMT), Funds of Science Technology Department of Zhejiang Province (Nos 2014C37101 and 2015C37035), Zhejiang Province Bureau of Health (No 2015ZA009), Funds of Science Technology Department of Zhejiang Province (No 2015C37035), Open Fund of Zhejiang Provincial Top Key Discipline of Biology. This work was supported by grants from the Healthy and Family Planning Commission of Zhejiang (No 2015-W105) to JS and the Zhejiang Provincial People's Hospital (No KYPT-2015) to JS.

\section{Disclosure}

The authors report no conflicts of interest in this work.

\section{References}

1. Kim D, Hoory T, Monie A, et al. Delivery of chemotherapeutic agents using drug-loaded irradiated tumor cells to treat murine ovarian tumors. J Biomed Sci. 2010;17:61. 
2. Cafferata EG, Podhajcer OL. Personalized virotherapy in cancer. Aging (Albany NY). 2015;7(5):288-289.

3. Rocconi RP, Zhu ZB, Stoff-Khalili M, et al. Treatment of ovarian cancer with a novel dual targeted conditionally replicative adenovirus (CRAd). Gynecol Oncol. 2007;105(1):113-121.

4. Kim KH, Dmitriev IP, Saddekni S, et al. A phase I clinical trial of Ad5/3-Delta24, a novel serotype-chimeric, infectivity-enhanced, conditionally-replicative adenovirus (CRAd), in patients with recurrent ovarian cancer. Gynecol Oncol. 2013;130(3):518-524.

5. Xu HN, Huang WD, Cai Y, et al. HCCS1-armed, quadruple-regulated oncolytic adenovirus specific for liver cancer as a cancer targeting gene-viro-therapy strategy. Mol Cancer. 2011;10:133.

6. Zhang Y, Gu J, Zhao L, et al. Complete elimination of colorectal tumor xenograft by combined manganese superoxide dismutase with tumor necrosis factor-related apoptosis-inducing ligand gene virotherapy. Cancer Res. 2006;66(8):4291-4298.

7. Oberley LW, Oberley TD. The role of superoxide dismutase and gene amplification in carcinogenesis. $J$ Theor Biol. 1984;106(3):403-422.

8. Behrend L, Mohr A, Dick T, Zwacka RM. Manganese superoxide dismutase induces p53-dependent senescence in colorectal cancer cells. Mol Cell Biol. 2005;25(17):7758-7769.

9. Weydert C, Roling B, Liu J, et al. Suppression of the malignant phenotype in human pancreatic cancer cells by the overexpression of manganese superoxide dismutase. Mol Cancer Ther. 2003;2(4):361-369.

10. Duan H, Zhang HJ, Yang JQ, Oberley LW, Futscher BW, Domann FE. MnSOD up-regulates maspin tumor suppressor gene expression in human breast and prostate cancer cells. Antioxid Redox Signal. 2003;5(5):677-688.

11. Pan QW, Zhong SY, Liu BS, et al. Enhanced sensitivity of hepatocellular carcinoma cells to chemotherapy with a Smac-armed oncolytic adenovirus. Acta Pharmacol Sin. 2007;28(12):1996-2004.

12. Pan Q, Liu B, Liu J, Cai R, Wang Y, Qian C. Synergistic induction of tumor cell death by combining cisplatin with an oncolytic adenovirus carrying TRAIL. Mol Cell Biochem. 2007;304(1-2):315-323.
13. Wu YM, Zhang KJ, Yue XT, et al. Enhancement of tumor cell death by combining cisplatin with an oncolytic adenovirus carrying MDA-7/ IL-24. Acta Pharmacol Sin. 2009;30(4):467-477.

14. Ma B, Wang Y, Zhou X, et al. Synergistic suppression effect on tumor growth of hepatocellular carcinoma by combining oncolytic adenovirus carrying XAF1 with cisplatin. J Cancer Res Clin Oncol. 2015;141(3):419-429.

15. Li J, Bonifati S, Hristov G, et al. Synergistic combination of valproic acid and oncolytic parvovirus $\mathrm{H}-1 \mathrm{PV}$ as a potential therapy against cervical and pancreatic carcinomas. EMBO Mol Med. 2013;5(10):1537-1555.

16. Kaiser J. Combining targeted drugs to stop resistant tumors. Science. 2011;331(6024):1542-1545.

17. Kruyt FA, Curiel DT. Toward a new generation of conditionally replicating adenoviruses: pairing tumor selectivity with maximal oncolysis. Hum Gene Ther. 2002;13(4):485-495.

18. Post DE, Khuri FR, Simons JW, Van Meir EG. Replicative oncolytic adenoviruses in multimodal cancer regimens. Hum Gene Ther. 2003;14(10):933-946.

19. Libertini S, Iacuzzo I, Ferraro A, et al. Lovastatin enhances the replication of the oncolytic adenovirus $\mathrm{d} 11520$ and its antineoplastic activity against anaplastic thyroid carcinoma cells. Endocrinology. 2007;148(11):5186-5194.

20. Pan JJ, Zhang SW, Chen CB, et al. Effect of recombinant adenovirusp53 combined with radiotherapy on long-term prognosis of advanced nasopharyngeal carcinoma. J Clin Oncol. 2009;27(5):799-804.

21. Fillastre JP, Raguenez-Viotte G. Cisplatin nephrotoxicity. Toxicol Lett. 1989;46(1-3):163-175.

22. Heise C, Sampson-Johannes A, Williams A, McCormick F, Von HoffDD, Kirn DH. ONYX-015, an E1B gene-attenuated adenovirus, causes tumor-specific cytolysis and antitumoral efficacy that can be augmented by standard chemotherapeutic agents. Nat Med. 1997;3(6):639-645.
OncoTargets and Therapy

\section{Publish your work in this journal}

OncoTargets and Therapy is an international, peer-reviewed, open access journal focusing on the pathological basis of all cancers, potential targets for therapy and treatment protocols employed to improve the management of cancer patients. The journal also focuses on the impact of management programs and new therapeutic agents and protocols on

\section{Dovepress}

patient perspectives such as quality of life, adherence and satisfaction The manuscript management system is completely online and includes a very quick and fair peer-review system, which is all easy to use. Visit http://www.dovepress.com/testimonials.php to read real quotes from published authors. 